\title{
Deformation behavior of Fe-based bulk metallic glass during nanoindentation
}

\author{
LI Lei ${ }^{1}$, LIU Yuan ${ }^{1}$, ZHANG TaiHua $^{2}$, GU JianSheng ${ }^{1}$ \& WEI BingChen ${ }^{1 \dagger}$ \\ ${ }^{1}$ National Microgravity Laboratory, Institute of Mechanics, Chinese Academy of Sciences, Beijing 100080 , \\ China; \\ ${ }^{2}$ State Key Laboratory of Nonlinear Mechanics (LNM), Institute of Mechanics, Chinese Academy of Sci- \\ ences, Beijing 100080, China
}

Fe-based bulk metallic glasses (BMGs) normally exhibit super high strength but significant brittleness at ambient temperature. Therefore, it is difficult to investigate the plastic deformation behavior and mechanism in these alloys through conventional tensile and compressive tests due to lack of distinct macroscopic plastic strain. In this work, the deformation behavior of $\mathrm{Fe}_{52} \mathrm{Cr}_{15} \mathrm{Mo}_{9} \mathrm{Er}_{3} \mathrm{C}_{15} \mathrm{~B}_{6} \mathrm{BMG}$ was investigated through instrumented nanoindentation and uniaxial compressive tests. The results show that serrated flow, the typical plastic deformation feature of BMGs, could not be found in as-cast and partially crystallized samples during nanoindentation. In addition, the deformation behavior and mechanical properties of the alloy are insensitive to the applied loading rate. The mechanism for the appearance of the peculiar deformation behavior in the Fe-based BMG is discussed in terms of the temporal and spatial characteristics of shear banding during nanoindentation.

bulk metallic glass, nanoindentation, serrated flow, shear band

\section{Introduction}

The ferromagnetic bulk metallic glasses (BMGs) have important potential applications in magnetic functional materials, and therefore, Fe-based BMGs have drawn increasing attention in metallic glass field since they were developed ${ }^{[1]}$. During the past decade, Fe-based BMGs with high glass forming ability (GFA) and excellent magnetic properties have been continually developed $\frac{[1-5]}{}$. On the other hand, non-ferromagnetic (paramagnetic at ambient temperature) Fe-based BMGs, such as "amorphous steel”, with a high GFA have been found recently ${ }^{[6-10]}$. This kind of Fe-based BMGs are potential structural materials due to their super high strength, low cost, and high GFA. However, most Fe-based BMGs are very brittle with the fracture toughness of only $1-2 \mathrm{MPa} \cdot \mathrm{m}^{1 / 2}$ and zero

Received April 2, 2007; accepted December 14, 2007

doi: 10.1007/s11433-008-0042-5

${ }^{\dagger}$ Corresponding author (email: weibc@imech.ac.cn)

Supported by the National Natural Science Foundation of China (Grant Nos. 50571109, 10572142 and 50771102) and the National Basic Research

Program of China (973 Program)(Grant No. 2007CB613900) 
compressive plastic strain ${ }^{[11]}$, though some samples exhibit a limited plasticity ${ }^{[3,9]}$. The lack of macroscopic plasticity in the Fe-based BMGs hinders the understanding of plastic deformation mechanism in these BMGs.

Recently, the instrumented nanoindentation method has been used to study the deformation behavior of BMGs more and more $\frac{[12-17]}{}$. During nanoindentation, the samples are in multi-axial stress state. The propagation of shear bands is restricted by the surrounding elastically deformed materials, and thus the rapid propagation of shear bands and their transition into cracks are retarded. Consequently, nanoindentation tests allow considerably larger plastic deformation to be accumulated in these quasi-brittle materials in a localized area around the indented regions. Furthermore, quite small volume of materials is needed for nanoindentation tests, which not only greatly reduces the effort for sample preparation but also reduces the sample for sample variation in property. Such factors facilitate observation of the mechanisms of plastic deformation under well-controlled conditions. Many recent results have shown that BMGs usually exhibit discrete strain (serrated flow) during nanoindentation, which is related to the formation and operation of shear bands. The serrated flow feature depends on the applied strain rate and the chemical composition of the alloy ${ }^{[12-17]}$. Most metallic glasses systems show a pronounced serrated flow at the low strain rates, while weak or no serrated flow at high strain rates. In contrast, the Ce-based BMGs with low glass transition temperature $\left(T_{\mathrm{g}}\right)$ exhibit a strong serrated flow only at high strain rates. Up to now, there is still little work reported on the plastic deformation behavior of BMGs with high $T_{\mathrm{g}}$ during nanoindentation. In this paper, the deformation behavior of $\mathrm{Fe}_{52} \mathrm{Cr}_{15} \mathrm{Mog}_{9} \mathrm{Er}_{3} \mathrm{C}_{15} \mathrm{~B}_{6}$ BMG with instinct brittleness and high $T_{\mathrm{g}}$ developed by Ponnambalam et al. was investigated through nanoindentation method ${ }^{[7,18]}$. The effect of partial crystallization on the mechanical behavior was also studied.

\section{Experimental methods}

$\mathrm{Fe}_{52} \mathrm{Cr}_{15} \mathrm{Mo}_{9} \mathrm{Er}_{3} \mathrm{C}_{15} \mathrm{~B}_{6}$ cylindrical rods with a diameter of $3 \mathrm{~mm}$ were prepared by arc-melting a mixture of elements with purity higher than $99.9 \%$ in an argon atmosphere and then chill-casting into a copper mould. The amorphous structure of the samples was confirmed by X-ray diffraction (XRD) using $\mathrm{Cu} \mathrm{K \alpha}$ radiation. Thermal analysis was performed with a Perkin-Elmer DSC-7 differential scanning calorimeter under argon atmosphere, at a constant heating rate of $20 \mathrm{~K} / \mathrm{min}$. Partially crystallized samples were obtained by annealing the BMG within a supercooled liquid region for different durations. The specimens for nanoindentation measurements were mechanically polished to a mirror finish and tested in a MTS Nano Indenter ${ }^{\circledR}$ XP with a Berkovich diamond tip. Fused silica was used as a reference sample for the initial tip calibration procedure. The indentations were performed in a load-control mode to a depth limit of $1 \mu \mathrm{m}$ using loading rates from 0.075 to $1 \mathrm{mN} / \mathrm{s}$. The thermal drift of the instrument was maintained below $0.05 \mathrm{~nm} / \mathrm{s}$. At least five indentations were made for each test at room temperature (296 K). Uniaxial compression tests were performed by an Instron 8562 testing device at an initial strain rate of $1.0 \times 10^{-4} \mathrm{~s}^{-1}$. The samples for compression tests are $6 \mathrm{~mm}$ in length and $3 \mathrm{~mm}$ in diameter. The morphology of indents after nanoindentation tests and fracture surface was observed by a JSM-6460 scanning electron microscope (SEM) and a Neophot-21 optical microscope. 


\section{Results and discussion}

Figure 1 shows the DSC curves of the as-cast $\mathrm{Fe}_{52} \mathrm{Cr}_{15} \mathrm{Mo}_{9} \mathrm{Er}_{3} \mathrm{C}_{15} \mathrm{~B}_{6} \mathrm{BMG}$ obtained at a constant heating rate of $20 \mathrm{~K} / \mathrm{min}$. It can be seen that the glass transition temperature $\left(T_{\mathrm{g}}\right)$, the crystallization temperature $\left(T_{\mathrm{x}}\right)$ and the supercooled liquid region $\left(\Delta T_{\mathrm{x}}=T_{\mathrm{x}}-T_{\mathrm{g}}\right)$ of the as-cast BMG are 810, 900 and $90 \mathrm{~K}$, respectively. These agree well with the reported results ${ }^{[7]}$. Partially crystallized samples are obtained by annealing the as-prepared BMG within the supercooled liquid region for different durations. The crystallization degrees are calculated by comparison of the enthalpy difference of exothermic peak between the crystallized samples and as-cast sample in the DSC measurements. The volume fraction of the crystallized phase for different partially crystallized samples ranges from $5 \%$ to $38 \%$. The DSC curves for the typical annealed samples with $5 \%$ and $18 \%$ crystallized phase are also exhibited in Figure 1. It can be seen that $T_{\mathrm{g}}$ of the alloy is insensitive to the partial crystallization, whereas $T_{\mathrm{x}}$ decreases with the increase of the crystallization degree, reflecting the decrease in the thermal stability of the residual amorphous phase in the partially crystallized samples.

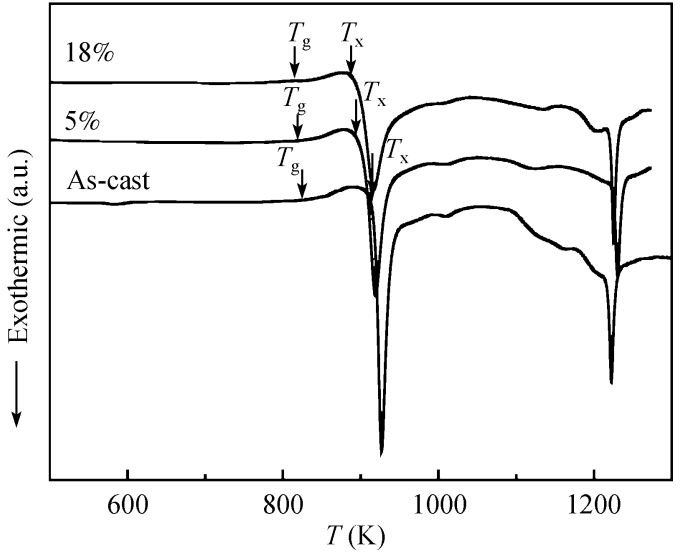

Figure 1 DSC curves for the as-cast and annealed $\mathrm{Fe}_{52} \mathrm{Cr}_{15} \mathrm{Mo}_{9} \mathrm{Er}_{3} \mathrm{C}_{15} \mathrm{~B}_{6}$ BMGs at a heating rate of $20 \mathrm{~K} / \mathrm{min}$. The numbers represent the content of crystallized phases.

The deformation behaviors of the as-cast $\mathrm{Fe}_{52} \mathrm{Cr}_{15} \mathrm{Mo}_{9} \mathrm{Er}_{3} \mathrm{C}_{15} \mathrm{~B}_{6} \mathrm{BMG}$ and partially crystallized samples are studied by nanoindentation. Figure 2 shows the typical load-displacement $(P-h)$ curves for the as-cast sample during nanoindentation at various applied loading rates (some curves are moved horizontally in order to distinguish each curve clearly). The results show that the hardness and the modulus of the alloy are insensitive to the applied loading rates. The measured average hardness and average modulus are $15.9 \mathrm{GPa}$ and $281 \mathrm{GPa}$, respectively. It can be seen that $\mathrm{Fe}_{52} \mathrm{Cr}_{15} \mathrm{Mo}_{9} \mathrm{Er}_{3} \mathrm{C}_{15} \mathrm{~B}_{6}$ BMG exhibits continuous plastic deformation at various applied loading rates during the loading process, and no discrete flow phenomenon (serrated flow) can be found even in the enlarged figure (inset in Figure 2). This characteristic is remarkably different with the deformation behavior of other BMGs during nanoindentation. Previous results have shown that Mg-, Zr-, and Pd-based BMGs exhibit strong serrated flow in the loading parts during nanoindentation, especially at the low applied loading rates $\stackrel{[12-17]}{ }$. The appearance of serrated flow is related to the formation and propagation of the shear bands during plastic deformation. During nanoindentation, when the local stress concentration reaches a certain extent, shear bands begin to form and propagate. The rapid propagation of shear bands induces the sudden release of elastic energy around the shear bands, thereby the visible discrete deformation is formed with the displacement bursts (pop-ins) under the load controlled condition. At low applied loading rates, the operation of single shear band is enough to accommodate the whole plastic deformation with the appearance of clear pop-ins in $P$ - $h$ curve; while at high applied rates, the disappearance of the pronounced serrated flow is supposed to be the simultaneous operation of multiple shear bands to accommodate the rapid plastic deformation $\frac{[12,13]}{}$. In present work, however, no pronounced serrated flow could be found for Fe-based BMG during the loading process even at a very low loading 
rate $(0.075 \mathrm{mN} / \mathrm{s})$. In order to understand the characteristics of shear band for Fe-based BMG during nanoindentation, plastic deformation features around the indents were studied through SEM.

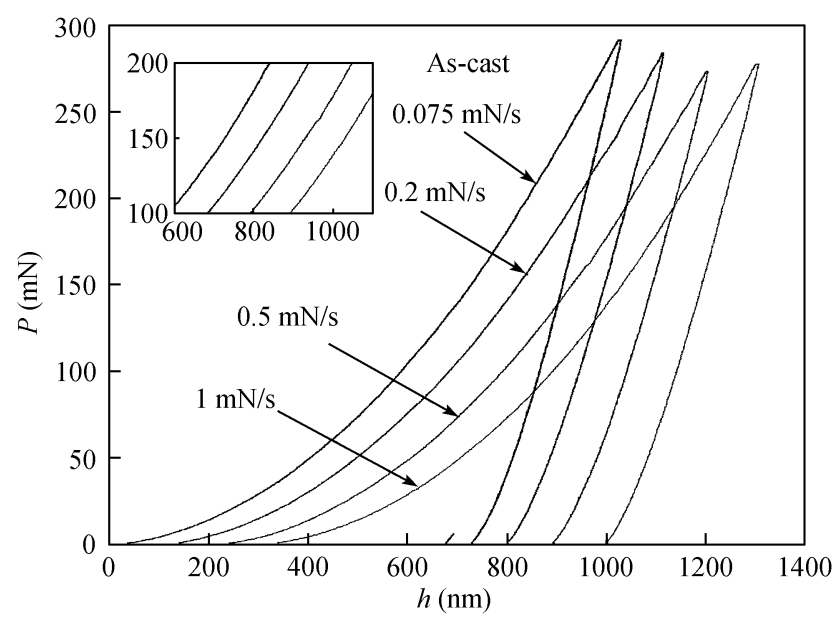

Figure 2 Typical load-displacement $(P-h)$ curves during nanoindentation at various loading rates for the as-cast sample. The inset is the enlarged parts of the loading curves.

Figure 3 shows the SEM images of typical surface deformation features around the indents after nanoindentation for $\mathrm{Fe}_{52} \mathrm{Cr}_{15} \mathrm{Mo}_{9} \mathrm{Er}_{3} \mathrm{C}_{15} \mathrm{~B}_{6} \mathrm{BMG}$ at the loading rate of $0.075 \mathrm{mN} / \mathrm{s}$ (Figure 3(a)) and $0.5 \mathrm{mN} / \mathrm{s}$ (Figure 3(b)). It reveals that distinct semicircle shear bands around the indents form around the indents after nanoindentation at all applied loading rates, while more shear bands form at the high loading rates. The shear band morphology and the correlation of shear band number with applied loading rates are consistent with that of other BMG systems ${ }^{[12,13]}$. This result indicates that although the alloy does not exhibit a pronounced serrated flow in the $P$-h curves (Figure 2) during nanoindentation, the formation and propagation of shear bands are the prominent characteristics for Fe-based BMG during the plastic deformation process. It is well-known that the presence of crystallized phase in the amorphous matrix significantly affects the formation and propagation of shear bands. Here we also examine the effect of partial crystallization on the deformation behavior of this alloy during nanoindentation.
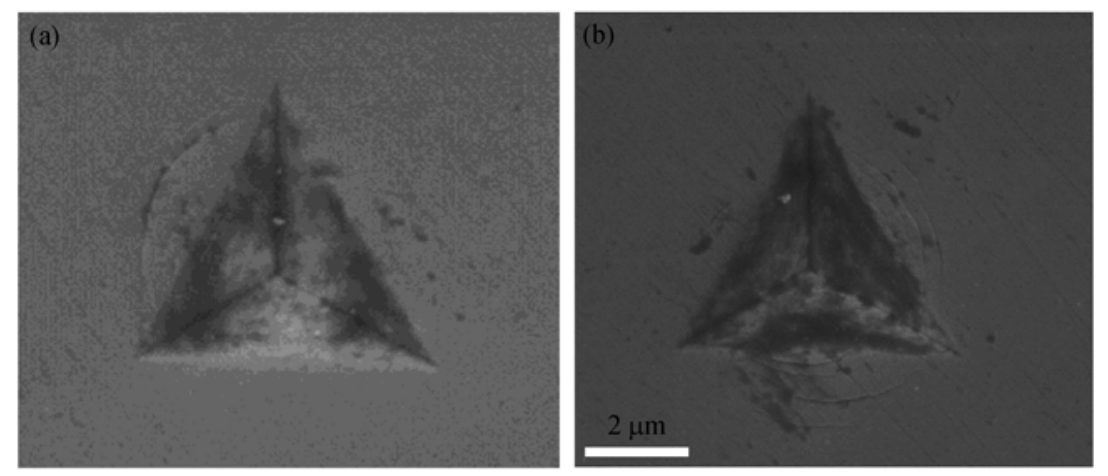

Figure 3 SEM images of the typical surface deformation features around the indents after nanoindentation at various loading rates: (a) $0.075 \mathrm{mN} / \mathrm{s}$; (b) $0.5 \mathrm{mN} / \mathrm{s}$. 
Figure 4(a) and (b) exhibits the typical load-displacement $(P-h)$ curves during nanoindentation at loading rates of $0.075-1.0 \mathrm{mN} / \mathrm{s}$ for $5 \%$ and $18 \%$ crystallized BMGs, respectively. The hardness and elastic modulus of the $5 \%$ crystallized sample are $16.2 \mathrm{GPa}$ and $299 \mathrm{GPa}$, respectively, which are $1.9 \%$ and $6.4 \%$, respectively, higher than those of the as-cast sample. As the crystallization degree increases to $18 \%$, the hardness of the alloy increases further, but the elastic modulus keeps a constant value. The values are $16.5 \mathrm{GPa}$ and $299 \mathrm{GPa}$, respectively, which are 3.8\% and $6.4 \%$ higher than those of the as-cast sample. It should be noted from Figure 4 that the partially crystallized Fe-based BMG also exhibits a smooth loading curve without distinct serrated flow at all the studied loading rates.
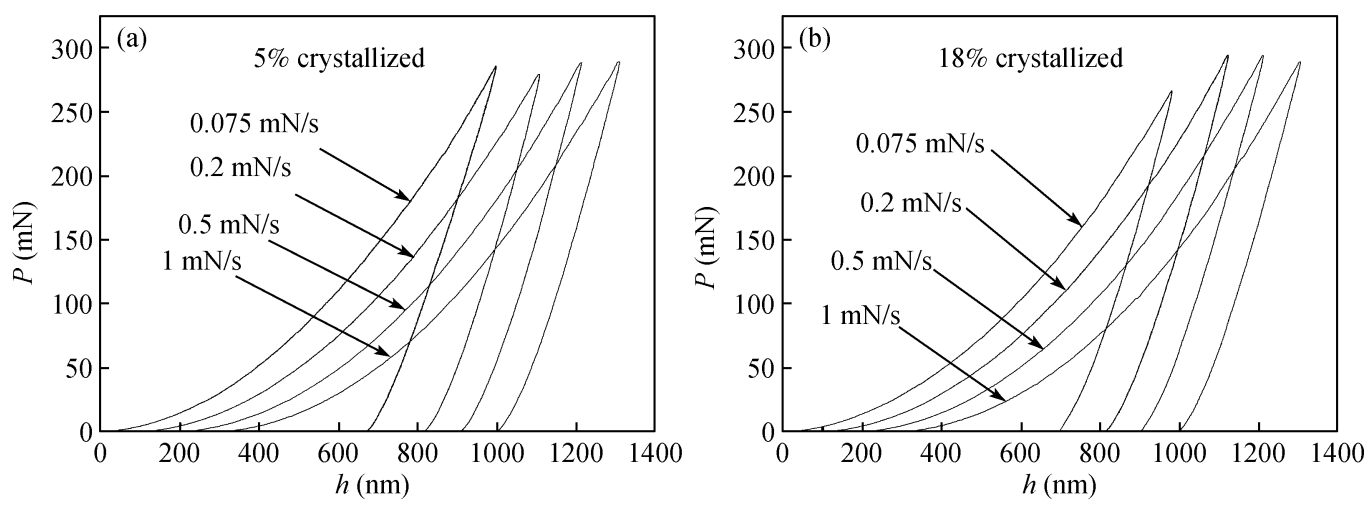

Figure 4 Typical load-displacement $(P-h)$ curves during nanoindentation at various loading rates for the partially crystallized samples: (a) $5 \%$ crystallized; (b) $18 \%$ crystallized.

For further understanding the deformation feature of the Fe-based BMG at ambient temperature, we investigated the compressive deformation behavior of the alloy through uniaxial compressive tests. Figure 5 shows the stress-strain curves for as-cast and partially crystallized samples at the initial strain rate of $1 \times 10^{-4} \mathrm{~s}^{-1}$. The studied samples failed immediately after elastic limit without distinct macroscopic plastic strain during the compressive test. The fracture strength $\left(\sigma_{\mathrm{b}}\right)$ and elastic strain of the as-cast sample are $3883 \mathrm{MPa}$ and 1.95\%, respectively. Figure 5 illuminates that $\sigma_{\mathrm{b}}$ increases with the crystallization degrees up to the crystallization content of $30 \%$. $\sigma_{\mathrm{b}}$ is 4467 MPa for the $30 \%$ crystallized alloy, which is $15.6 \%$ higher than that of the as-cast sample. The tendency that $\sigma_{\mathrm{b}}$ is increasing with the content of crystalline phases is consistent with the above relationship between the hardness values and crystallization degrees. However, it should be noted that annealing induced crystallization normally leads to the increase of fragility of BMGs, caused by the precipitation of brittle intermetallic phases. Here, partial crystallization increases $\sigma_{\mathrm{b}}$ of the alloy, which indicates that the crystallization does not induce the premature failure of the material. In contrast, the presence of fine crystallized phases may suppress the rapid propagation of a single shear band. Figure 6 shows the SEM images of the typical surface deformation features around the indents of the $5 \%$ crystallized sample after indentation at the loading rate of $0.075 \mathrm{mN} / \mathrm{s}$. It can be seen that the shear bands number in the crystallized alloy is slightly larger than that in the as-cast alloy at the same loading rate. Moreover, the shear band upset scale of the shear bands is much smaller in the partially crystallized alloy at the same loading rate. This indicates that the rapid propagation of a single shear band is suppressed in the partially crystallized alloys. 


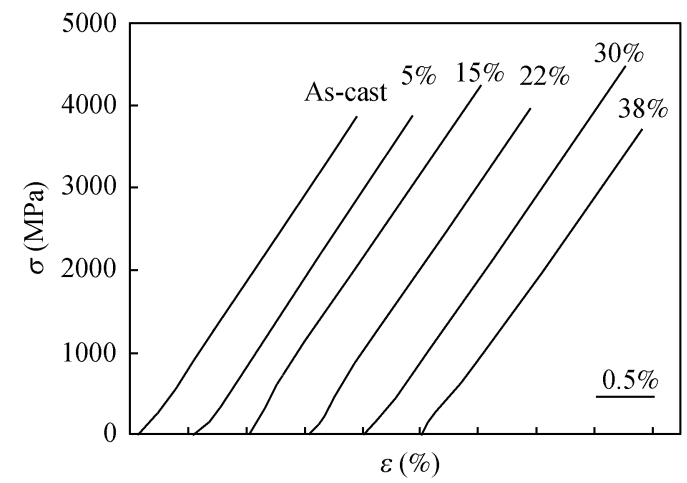

Figure 5 Stress-strain curves for as-cast and partially crystallized samples during uinaxial compression. The numbers illuminate the volume fraction of crystalline phases.

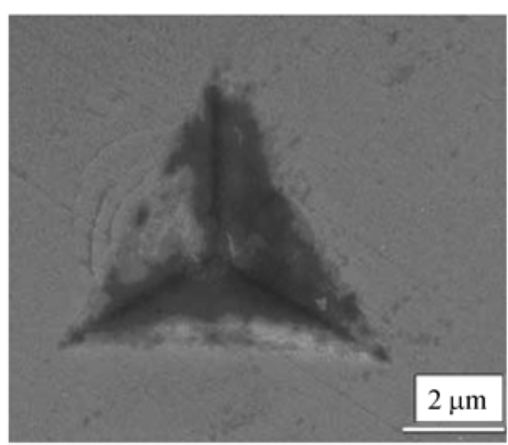

Figure 6 SEM images of the typical surface deformation features around the indents for $5 \%$ crystallized alloy after nanoindentation at $0.075 \mathrm{mN} / \mathrm{s}$.

Schuh et al. have done many researches on the relationship between the serrated flow phenomenon and the loading rates during nanoindentation for Zr-, Pd-, and Mg-based BMGs. It was proposed that the weakening of serrated flow at high strain rates is correlated with the simultaneous operation of multiple shear bands. Moreover, the nucleation of shear bands may be suppressed at high enough strain rates due to the limited time scale, giving rise to a macroscopically homogeneous deformation. However, in present case, shear band features indicate that shear band number does not show a remarkable increase with the loading rate so that the transition of deformation mode from inhomogeneous to homogeneous deformation could occur. Recently, Nieh et al. suggested that the number density of shear bands increased at a low normalized temperature $\left(T / T_{\mathrm{g}}, T\right.$ is the measured temperature) by using a linear instability theory ${ }^{[17]}$. This agrees with the experimental results of instrumented macroindentation on various BMG systems, wherein the shear bands number beneath the indents for the alloy with high $T_{\mathrm{g}}$ (for example Ni-based BMG) is much higher than that of the alloy with low $T_{\mathrm{g}}$ at the same strain rate at the ambient temperature $\frac{[19]}{}$. This suggests that although high number density of shear bands is not observed on the upper surface of the indents, a great number of fine shear bands may exist underneath the indents after nanoindentation. In addition, according to the shear transformation zone (STZ) model ${ }^{[20,21]}$, the ratio of the shear strain rate inside a STZ to that of the surrounding materials is also correlated with the normalized temperature. At low normalized temperatures (i.e. the present Fe-based BMG with high $T_{\mathrm{g}}$ and tested at ambient temperature), the local shear strain rates inside STZ are higher by about 5 magnitudes than that of the surrounding material. This means that the local strain rates inside a shear band for Fe-based BMG at the ambient temperature are extremely high. On the other hand, the number density of shear bands in the present BMG is much higher than that in other BMG systems with relatively lower $T_{\mathrm{g}}$. These two factors contribute to a much smaller temporal and spatial scale of shear banding in the present Fe-based BMG, compared with that of Zr-, Pd-, and Mg-based BMGs. Under this condition, the sudden release of elastic energy around the shear band caused by the propagation of shear band is difficult to be detected by the nanoindentation equipment, so that the macroscopically continuous plastic deformation occurs. Furthermore, the spatial scale of shear band operation is suppressed further by the presence of crystalline phases, and thereby the serrated flow cannot be found in partially crystallized samples at various loading rates either. 


\section{Summary}

The plastic deformation behavior and shear banding feature in $\mathrm{Fe}_{52} \mathrm{Cr}_{15} \mathrm{Mog}_{9} \mathrm{Er}_{3} \mathrm{C}_{15} \mathrm{~B}_{6} \mathrm{BMG}$ with high glass transition temperature were studied through the instrumented nanoindentation and compressive test. Partial crystallization (less than 30\%) improves the fracture strength and hardness of the alloy. During the loading process of nanoindentation, the amorphous and partially crystallized alloys all exhibit a smooth plastic deformation feature during nanoindentation at all the studied loading rates, though a distinct shear band pattern can be found around the indents after indentation tests. The absence of serrated flow during plastic deformation in the Fe-based alloy is correlated with the quite small temporal and spatial scale of shear banding.

1 Inoue A, Zhang T, Takeuchi A. Bulk amorphous alloys with high mechanical strength and good soft magnetic properties in Fe-TM-B (TM = IV - VIII group transition metal) system. Appl Phys Lett, 1997, 71: 464-466[DOI]

2 Pang S J, Zhang T, Asami K, et al. Synthesis of Fe-Cr-Mo-C-B-P bulk metallic glasses with high corrosion resistance. Acta Mater, 2002, 50: 489-497[DOI]

3 Shen B L, Akiba M, Inoue A. Excellent soft-ferromagnetic bulk glassy alloys with high saturation magnetization. Appl Phys Lett, 2006, 88: 131907[DOI]

4 Chang C T, Shen B L, Inoue A. FeNi-based bulk glassy alloys with superhigh mechanical strength and excellent soft-magnetic properties. Appl Phys Lett, 2006, 89: 051912[DOI]

$5 \mathrm{Hu} \mathrm{Y,} \mathrm{Liu} \mathrm{L,} \mathrm{Chan} \mathrm{K} \mathrm{C,} \mathrm{et} \mathrm{al.} \mathrm{The} \mathrm{effect} \mathrm{of} \mathrm{crystallization} \mathrm{on} \mathrm{microstructure} \mathrm{and} \mathrm{magnetic} \mathrm{properties} \mathrm{of}$ $\mathrm{Fe}_{61} \mathrm{Co}_{7} \mathrm{Zr}_{9.5} \mathrm{Mo}_{5} \mathrm{~W}_{2} \mathrm{~B}_{15.5}$ bulk metallic glass. Mater Lett, 2006, 60: 1080-1084[DOI]

6 Lu Z P, Liu C T, Thompson J R, et al. Structural amorphous steels. Phys Rev Lett, 2004, 92: 245503[DOI]

7 Ponnambalam V, Poon S J, Shiflet G J. Fe-based bulk metallic glasses with diameter thickness larger than one centimeter. J Mater Res, 2004, 19: 1320-1323[DOI]

8 Shen J, Chen Q J, Sun J F, et al. Exceptionally high glass-forming ability of an FeCoCrMoCBY alloy. Appl Phys Lett, 2005, 86: 151907[DOI]

9 Yao K F, Zhang C Q. Fe-based bulk metallic glass with high plasticity. Appl Phys Lett, 2007, 90: 061901[DOI]

10 Gu X J, McDermott A G, Poon S J, et al. Critical poisson's ratio for plasticity in Fe-Mo-C-B-Ln bulk amorphous steel. Appl Phys Lett, 2006, 88: 211905[DOI]

11 Wang W H, Dong C, Shek C H. Bulk metallic glasses. Mater Sci Eng R, 2004, 44: 45-89[DOI]

12 Schuh C A, Lund A C, Nieh T G. New regime of homogeneous flow in the deformation map of metallic glasses. Acta Mater, 2004, 52: 5879-5891[DOI]

13 Schuh C A, Nieh T G. A nanoindentation study of serrated flow in bulk metallic glasses. Acta Mater, 2003, 51: 87-99 [DOI]

14 Xing D M, Zhang T H, Li W H, et al. Characterization of plastic flow in two Zr-based bulk metallic glasses. Sci China Ser E-Technol Sci, 2006, 49: 414-424[DOI]

15 Wei B C, Zhang L C, Zhang T H, et al. Strain rate dependence of plastic flow in Ce-based bulk metallic glass during nanoindentation. J Mater Res, 2007, 22: 258-263[DOI]

16 Dai L H, Liu L F, Yan M, et al. Serrated plastic flow in a Zr-based bulk metallic glass during nanoindentation. Chin Phys Lett, 2004, 21: 1593-1595[DOI]

17 Gao Y F, Yang B, Nieh T G. Thermomechanical instability analysis of inhomogeneous deformation in amorphous alloys. Acta Mater, 2007, 55: 2319 -2327[DOI]

18 Lewandowski J J, Wang W H, Greer A L. Intrinsic plasticity or brittleness of metallic glasses. Philo Mag Lett, 2005, 85: 77-87[DOI]

19 Li W H, Zhang T H, Xing D M, et al. Instrumented indentation study of plastic deformation in bulk metallic glasses. J Mater Res, 2006, 21: 75-81[DOI]

20 Argon A S. Plastic deformation in metallic glasses. Acta Metall, 1979, 27: 47-58[DOI]

21 Falk M L. Molecular-dynamics study of ductile and brittle fracture in model noncrystalline solids. Phys Rev B, 1999, 60: 7062-7070[DOI] 